\title{
Plant parts: processes, structures, or functions?
}

\author{
D.A. Baum \\ Department of Botany, University of Wisconsin, \\ 430 Lincoln Drive, Madison WI 53706, USA \\ dbaum@wisc.edu
}

"...it has become increasingly accepted that in plants it is better to consider an organism and what are perceived by many as its component parts at any one time as a snapshot in a dynamic transformation of growth, maturity and senescence..."

— Mabberley \& Hay, 1994 : 122.

\begin{abstract}
It is usually taken for granted that plants are composed of a series of discrete parts that can readily be compared both within a single plant and between closely or even distantly related organisms. The biological meaning of 'part' needs to be made more rigorous such that the naming of a part or the homologising of parts among organisms constitutes a scientific hypothesis that is testable, at least in principle. I explore three alternative approaches to defining parts. The parts-as-structures approach holds that parts (or "phenes") are aspects of an organism that would not have been formed during development in the absence of causal genetic factors. A structural phene hypothesis is refuted by the lack of a hypothetical genetic deletion that would ablate just this part of the organism. The parts-as-functions approach focuses on pieces of the organism that could have been functionally different if one or more gene in the genome were mutated. A functional phene hypothesis is refuted by showing that all mutations affecting the phene have pleiotropic consequences. The part-as-process approach equates parts with developmental modules, making it especially helpful for addressing the concept of serial homology. I conclude that, depending on the context, parts are best understood sometimes as structures, sometimes as functions, and sometimes as processes, but in each case we need to develop rigorous concepts rather than falling back on human perception as the ultimate arbiter of part-ness.
\end{abstract}

Keywords. Atomisation, developmental causation, evo-devo, homology, phenes, process morphology, serial homology

\section{Introduction}

At least since Goethe, botanists have seen vascular plants, or at least their aboveground portions, as a series of distinct and repeated parts - leaves, stems, flowers, fruits, seeds, etc. Insofar as these parts are readily recognisable and assignable to familiar umbrella categories, as they usually are, they serve as landmarks that allow a knowledgeable observer to infer aspects of the plant's growth history. For example, it is usually obvious which leaves or shoots are younger and which are older and where growth has been interrupted by extrinsic damage. Likewise, the comparison of plant 
parts among species is the basis for all aspects of taxonomy, from field identification, to species designation, to morphological phylogenetic analysis (though the latter is increasingly rare except in palaeobotany). In short, it is almost impossible not to look at a single plant and see it as a collection of parts and, moreover, to perceive that the same types of parts are to be found in other species, though perhaps with a different form or architectural arrangement. The same is probably true of all organisms, but I will focus my attention here only on plants and explore the key question, what are plant parts?

In a volume honouring my undergraduate tutor, David Mabberley, it seems appropriate to use as a jumping-off point, Hay \& Mabberley (1994). This paper, like other papers by these authors (Hay \& Mabberley, 1991; Mabberley \& Hay, 1994; Hay, 2019 in this volume), challenges the instinct to fragment a plant into parts. Instead, it argues for 'process morphology', an outlook that downplays the idea that organisms have discrete parts assignable to one of a few discrete flavours in favour of an emphasis on the dynamic nature of development. This more dynamical perspective is generally traced to Agnes Arber's influential book, The natural philosophy of plant form (Arber, 1950), but was fleshed-out in modern form by Rolf Sattler (e.g. Sattler, 1984, 1988, 1990, 1992, 1996), Rolf Rutishauser (Rutishauser, 1995, 1997, 1999; Rutishauser \& Isler, 2001; Sattler \& Rutishauser, 1990, 1997), and a few others (Kirchoff et al., 2008; Lacroix et al., 2003). The process morphology approach emerged in part as a counterpoint to the essentialism of some classical morphologists, most notably Troll (see Claßen-Bockhoff, 2001). In its purest form essentialistic morphology implied that leaves, shoots, and the like are fundamental building blocks of the plant archetype: the starting point (in the ideal sense rather than the modern temporal/evolutionary sense) from which all plants are derived. Essentialism implies that parts of a few more or less universal types are the conserved building blocks of all vascular plants.

Process morphologists challenge essentialistic morphology by questioning the underlying philosophy and by highlighting cases that defy discrete and unambiguous assignment of parts to types. There are, in fact, many oddball plants with morphological structures that seem impossible to adequately describe within Goethean morphology. When I was a student, Mabberley had me read about 'phyllomorphs', the strange leaf-stem intermediates of some Gesneriaceae, most famously Streptocarpus (Jong \& Burtt, 1975), and he was clearly tickled by the indeterminate pinnate 'leaves' (or are they shoots?) of some Meliaceae (e.g. Chisocheton; Mabberley, 1979). Other proponents of process morphology have focused on aquatic plants, such as Podostemaceae (Rutishauser, 1995, 1997, 2016) or Utricularia (Sattler \& Rutishauser, 1990; Rutishauser \& Isler, 2001; Rutishauser, 2016). These plants certainly have rules governing their development, and do seem to produce organs of some kind, but some of these organs do not have obvious one-to-one homologues in more canonical angiosperms suggesting, at best, a degree of partial or mixed homology (Sattler, 1984, 1996).

One manifestation of partial/mixed homology is another phenomenon that Mabberley had his students learn about, 'transference of function'. As first pointed out by Mabberley's mentor, John Corner, in his memorable 1958 paper (Corner, 1958), 
the ecological functions of plant parts are often transferred from one organ to another. Notably many of the examples Corner gave seem to imply the migration from one kind of organ to another, not just of an ecological function, but also of genetically encoded attributes, for example colour or fleshiness. Such cases of transference of function entail partial transfer of organ identity from one part of the plant to another, also called homeoheterotopy (Baum \& Donoghue, 2002). This can be understood as yielding an organ with mixed homology, something that would be disallowed in the most essentialistic approaches to plant morphology. Thus, the natural history of Corner provides a rich set of examples that support the agenda of process morphology over the more rigid categorisation that has historically dominated botany.

A final line of thought that supports the tenets of process morphology comes from the attempt to visualise plant form in all four dimensions. As eloquently described by Hay \& Mabberley (1994), organisms are dynamical systems in constant flux - processes not static structures - meaning that 'parts' are not sharply defined things like the nuts and bolts and gears of a car, but repeating eddies or vortices in developmental flows. Such a vision is helpful and explains some important aspects of plant parts. For example, like a vortex in a fluid, parts have inherently fuzzy edges and no two vortices are exactly alike even if generated by the same physical laws. Thus, considering plant development we have very little reason to believe that plant parts could be rigidly bounded things that can easily be assigned to discrete and general organ types.

What is fascinating, I think, is that for all the scientific and aesthetic appeal of process morphology biologists still catalogue, describe, and compare plants in terms of parts assigned to general categories; "fragment morphology" (Hay \& Mabberley, 1994). This will be obvious from any flora, dichotomous key, or introductory botany class. Why should this be so? I believe the apparent failure of botanists to internalise process morphology arises because this philosophy requires habits of thought and ways of communicating that are not natural for science in general (Seibt, 2012). Due to these possibly hard-wired psychological biases, it is very hard to resist the tendency to perceive plants as objects, drawing us to a language of nouns not verbs (Hay, 2019 in this volume). Thus, absent a new way of talking about plants, even the most processminded botanist is constrained to refer to plant fragments as though they were nuts or bolts or gears.

One response to this situation is to conclude that communication in plant morphology is entirely about subjective interpretation: more like a review of a piece of art than a scientific description of the natural world. Such an intersubjective viewpoint was described by Hay \& Mabberley (1994). This position is certainly defensible, but as they would agree, not entirely satisfying. As an alternative, I lean towards at least trying to define plant parts as entities that exist independent of human perception. Specifically, I think we should aim to define 'part' in such a way that pointing at a 'part' constitutes the posing of a scientific hypothesis - a statement of how the world actually is, irrespective of the impossibility of ever gaining perfect knowledge of the world as it is. It is this latter agenda that I will try and advance here. Instead of abandoning part-thinking, or replacing it with a fully intersubjective perspective, my 
goal is to firm up our understanding of how non-linear, fluid development processes can nonetheless yield organisms that are composed of parts that have boundaries, albeit fuzzy ones, that can meaningfully be compared between organisms of the same or different species.

The strategy I propose entails asking how we might fragment organisms into non-arbitrary parts in the case that we had God-like knowledge of all relevant genetic, developmental, and evolutionary processes. This is a good question to ask not because we will ever have such omniscience, but because progress in science often occurs from such a framing. As a case in point, think about how far systematics has come since Hennig, Zimmermann, and others articulated the view that only monophyletic or exclusive groups of organisms (i.e. those that are all more closely related to each other than to any organism outside the group) deserve recognition in taxonomy. By stating this desideratum clearly, these pioneers of phylogenetic systematics shifted the field to one where naming a taxon is interpreted as a hypothesis of exclusivity, which can then be critically evaluated using the best available scientific tools. To be sure, we can never establish patterns of common ancestry (i.e. relatedness) beyond any doubt, but that is true for any claim in empirical science. The shift in taxonomy from viewing taxa as percepts - groups of organisms recognised as taxa by an 'expert' - to viewing them as hypotheses of exclusivity has been a great advance that I would credit for the phylogenetic revolution that swept systematics starting in the 1980s. We may wonder, therefore, whether such a leap forward is possible for comparative morphology. Can we get to a point where describing or homologising a plant part can be viewed as a hypothesis about development or evolution rather than simply a statement of what a certain expert perceives?

To advance this goal I will lay out some alternative ways to understand the nature of plant parts such that instances of these parts can be said to be 'real', independent of whether they are observed or analysed by humans. I will start by focusing on parts as physical subcomponents of a plant body and describe two ways in which a plant part can be viewed as a scientific hypothesis: (1) parts as products of development, which I will call 'structures', and (2) parts as potential targets of selection, which I will call 'functions'. As the reader will see, both approaches entail fuzzy edges and imply that it will often not be possible to associate a part of one organism with one and only one homologue in a second organism - all very much in line with process morphology. Following this exploration of parts as physical components, I will explore an alternative approach, (3) dividing a developmental programme into meaningful subprogrammes, which I will call 'processes'. I will argue that for all its appeal, this last strategy is not very useful for making comparisons between organisms. Nonetheless, the notion of parts as processes is probably required as a way to make sense of serial homology, the perception that multiple parts within a single organism (e.g. leaves) are iterations of the same general thing. 


\section{Overview of the problem}

When botanists observe a plant and 'see' a set of parts with familiar names - leaves, flowers, or seeds, for example - they are making two mental leaps. First, they are intuiting that these parts have some kind of objective reality as meaningful subsets of the whole organism. Second, they are assuming that there is some notion of sameness that makes all the leaves on a plant equivalent to one another and to the 'leaves' of a different, perhaps distantly related plant. The questions of part existence (which subsets of an organism are valid parts) and part homology (what can justify equating 'leaves' between two organisms, or between two instances within the same organism) need to be carefully distinguished.

The problem of part existence is logically prior to homology (Baum, 2013; Olson, 2019). If, for example, parts were just subjective percepts without any existence outside of human perception then evaluating whether two 'leaves' are homologous would become a question of perceptual psychology rather than just natural science. Botanists should want more than that. At the very least we want it to be possible that somebody could be wrong when they claim that such and such is a part, even before they go on to claim that it is a leaf, or a flower, or a seed. To achieve this goal we need to articulate objective criteria for part individuation.

Although I have argued for keeping in mind that there are two issues, not one, they are nonetheless connected issues. In developing a conceptual understanding of part individuation, it is desirable that the units identified are potentially comparable across organisms - at least between parents and their offspring. This constraint suggests that our criterion of individuation should allow us to associate parts with heritable information, which is to say genomic features that are passed on from parents to offspring. Absent such a connection to heredity the idea of sameness of parts, even among close kin, would be untenable.

Here I will start by laying out a parts-as-structures approach, which is based on connecting aspects of the phenotype to their underlying genetic causes. Since I have previously explored this developmental-causal framework in some detail (Baum, 2013), I will only provide sufficient detail here to explain how it may be adapted into a parts-as-function version, which is to say one which looks not at developmental cause but at adaptive potential.

\section{Parts-as-structures: linking structures to their genetic causes}

As I have laid out previously (Baum, 2013), we can understand parts, or phenes, as those aspects of a phenotype that have some genetic cause. This means that for all valid parts, there is some subset of the genome whose removal would, counterfactually, result in an organism missing this and only this part of the organism. Thus, the hypothesis that a certain piece of an organism is a valid part is tested (in principle) by assessing whether there is some set of hypothetical deletions that could obliterate this part (and just this part) from the organism. While there is some unavoidable fuzziness 
due to developmental noise, with complete knowledge of the genotype - phenotype map (something that may not be possible in reality) parts could be delimited in a nonarbitrary way (Baum, 2013).

A key hypothesis (much in need of testing) is that many familiar plant parts, for example leaves and flowers, do not have a single genetic cause but are causally dependent on multiple genetic factors. Since there are many genetic changes that could, counterfactually, remove leaves or flowers, these structures are 'multiply-determined'. However, since the boundaries established by different genetic factors may be very similar yet still slightly different, some fuzziness regarding the limits of each part is implied.

Since this definition connects phenes to causal genetic factors, a structural phene can be said to be inherited from parents to offspring when its causal genetic factors are passed on. In the event that a phene is multiply-determined it is possible for genetic factors that cause the same phene in one generation to cause different phenes in the next generation. I have laid out three ways of handling such changes in genephene mapping, of which I will mention just one here, 'consensus homology' (Baum, 2013). This approach asserts that a multiply-caused parental phene is homologous to whichever phene in the offspring is dependent on the greatest number of shared (orthologous) genetic factors.

This structural notion of homology can readily be extended from parentoffspring to interspecific comparisons. In this case a phene in two different species is homologous if and only if all the ancestral parent - offspring pairs linking those species (and their phenes) to their common ancestor had homologous phenes. Because the basis of parent - offspring homology is the majority of the causal genes (consensus homology), the genetic causes of parts can change over time to the point that two species could share homologous phenes that are causally dependent on completely different genetic factors, a phenomenon sometimes called developmental systems drift (Haag, 2007; True \& Haag, 2001). In cases where a significant minority of genetic factors come to move their domain of causal efficacy, whether at one time or gradually over many generations, consensus homology also allows for partial homology (Sattler, 1996) via homeoheterotopy (Baum \& Donoghue, 2002).

\section{Parts-as-functions: linking traits to responses to selection}

The parts-as-structures approach connects phenes to those parts of the genome upon which the phene (and just the phene) depends. A parts-as-functions approach would, instead, attach phenes to those parts of the genome that could potentially mutate to alter the phene (and just the phene). If the parts-as-structures approach identifies the phenotypic products of the current developmental programme, whose structure has been shaped by historical natural selection, a parts-as-function approach identified those aspects of a phenotype that can be shaped by ongoing and future natural selection (e.g. Olson, 2019). In the same way that species individuation can be approached either retrospectively using a genealogical concept or prospectively by focusing on 
the potential for future gene exchange (e.g. Baum, 1998; Ereshefsky, 1992), it makes sense to develop an approach to trait individuation and homology that focuses on the potential for future adaptation. A structural phene, like a phylospecies, is a product of evolution ("phylon" sensu Reydon, 2005), whereas as a a functional phene, like a biospecies, is a player in evolution ("evolveron" sensu Reydon, 2005).

A key feature of a functional phene that predicts its capacity to undergo adaptive evolution for new functions is some degree of autonomy from the rest of the organism (Olson, 2019; Raff \& Raff, 2000; Wagner, 1989; Wagner \& Altenberg, 1996). It has long been appreciated that development modularity (e.g. Bonner, 1988; Gould, 1977) is important because it allows derived alleles to affect one part of the organism without pleiotropic effects on other parts. Thus, our approach to functional phene delimitation needs to consider developmental autonomy as its primary criterion.

In contrast to the part-as-structure approach, which asks, "Are there any genetic factors whose absence would have resulted in the absence of this part and no other part of the organism?", a part-as-function approach asks: "Are there any plausible mutations that could alter the functionality of this part of the organism without also altering the functionality of any other part of the organism?" When the organism is a member of a sexual population, this question can be addressed empirically by evaluating the genetic covariance of all measurable phenotypic traits in that population (Cheverud, 1984) or by seeing if directional selection can act independently on different traits (e.g. Beldade et al., 2002). However, while perhaps the most practical procedures, these approaches are incomplete since they only deal with allelic variation already in a population, not with potential future mutations. Creative combinations of quantitative studies within populations as well as cross-taxon comparisons can sometimes shed light on particular cases (e.g. Olson \& Rosell, 2006). However, we should recognise that evaluating part autonomy in practice will always be a very challenging endeavour.

The difficulty of empirically determining whether an aspect of the phenotype is or is not a functional phene in a particular organism or population does not undermine the part-as-function conception (any more than the practical challenges of delimiting structural phenes kills the parts-as-structures approach). So, let us take as a given that we somehow know all plausible mutational edits of our target genome and know which phenotypic alterations could potentially occur. For example, suppose that we establish that mutations are available to render perceptual leaves (all the leaves of a single plant but nothing else) longer or shorter, thicker or thinner, more or less hirsute, etc. and can point to the genes that would be altered by these mutations. These genes can be said to individuate the functional phene, 'leaves'.

Even if 'leaves' in a certain plant can be individuated based on developmental causation (part-as-structure) and on evolutionary potential (part-as-function), the prospective and retrospective entities would not be exactly the same thing. Despite being two sides of the same causal process, they will not always agree. The same is true of phylospecies and biospecies: an evolving population held together by gene flow, a biospecies, even if not currently exclusive, will tend to generate phylospecies, exclusive groups, in the future. Likewise, when selection acting on parts-as-functions causes fixation of derived alleles (e.g. those promoting drip-tips) the resulting rewiring 
of development may tend to cause new parts-as-structures to emerge (e.g. the drip-tip itself). Since there is certainly no clean symmetry or predictability in evolution in either the realms of species or part delimitation, we have a good reason to distinguish both prospective (biospecies, parts-as-processes) and retrospective (phylospecies, parts-as-structures) concepts depending on the scientific context.

Once functional phenes have been individuated based on those genes that can be targeted by selection, it is easy to see how functional phenes are inherited. The approach exactly mirrors the parts-as-structures framework, the only difference being that the list of genes individuating, say, leaves is different in each case: either genes whose absence would result in a lack of leaves (structural phene), or genes that could plausibly mutate to generate leaves with different features (functional phene). In each case, we can apply consensus homology to decide which structures in the next generation are homologous to the 'leaves' in this generation and can consider 'leaves' in two different species homologous if they are connected by repeated parent-offspring homology back through their common ancestor. As with the parts-as-structures approach, a parts-as-function approach would tend to delimit parts with fuzzy edges, since pleiotropy will not always drop to zero for all genes at the same spatial location. Likewise, partial homology and developmental-systems drift are possible as genes' pleiotropic potentials evolve.

\section{Parts-as-processes: the basis of serial homology}

The parts-as-structures and parts-as-functions approaches share in common that neither supports the serial homology of multiple parts within a single organism, something that is widely perceived in vascular plants, and also many animals (Minelli, 2003; Rutishauser, 2018). Both approaches allow that a certain species has a part 'leaves', and that this part may be homologous to the 'leaves' of a second species. However, neither allows a way to parse the question: Are the first and third leaves of this plant homologous? These 'different' leaves are really just discontinuous pieces of a single (structurally or functionally) individuated phene. Since we have been connecting phenes to features of the genome, and since all parts of a single plant share the same genome, there is no way to conclude serial homology under either a parts-as-structures and parts-as-functions approach. Our perception that a plant is composed of multiple distinct leaves rather than a single entity is incompatible with either the structural or functional approach just described. And the same is true for overlapping perceived modules, such phytomers, which include a portion of stem, an axillary bud, and a single leaf (White, 1979).

Given this incompatibility we need a different way to understand "part" that respects the fact that when we compare plants of different species our attention is drawn first to the similarities and differences of individual leaves (e.g. different shape, size, or indumentum) and only later to the totality of leaves e.g. different numbers of leaves). Thus, we should supplement the structural and functional approaches with a notion of serial homology that allows us to evaluate the truth of statements such as: 
"here we have two leaves, not one" or "leaf number 9 is more highly compound than leaf number 3."

Frameworks that looks at homology between individuals cannot readily 'see' serial homology because they are constrained to attach phenes to features of the genome. What we need, therefore, is to consider the more complicated question of how a genome is decoded into a phenotype: the unfolding of plant development. The almost miraculous process of development occurs when a genome, whose composition is usually identical across all cells, is subjected to context-dependent gene-regulation. Development, therefore, resembles a computer programme, albeit a special kind of programme that can, once booted in a zygote (sporophyte) or spore (gametophyte), build its own processors (new cells) as it proceeds. The developmental computer programme includes diverse do-while loops and the like, all encoded via transcriptional, translational, and post-translational regulation.

It has now come to be agreed that most successful groups of organisms show modularity (Wagner \& Altenberg, 1996), meaning that their developmental programme is composed of multiple semiautonomous subroutines, or gene-regulatory networks (GRN: Wagner, 2007). The modularity of developmental programmes is the underlying basis of the parts-as-processes approach, but here we want to go one step further and ask whether it allows us to recognise each perceptual leaf as a distinct process, a partas-process?

Insofar as each GRN defines two states (on vs. off), or perhaps a small number of discrete states, the intersection of the many GRNs in a developmental programme serves to define a finite number of discrete epigenetic states that cells can occupy. During development cells transition among these epigenetic states, with transitions occurring as a function of a cell's current state combined with signals received from other cells or the outside environment. Imagine watching the entirety of development, while seeing each cell coloured by its current epigenetic state: you would see a kaleidoscope of waves, spirals, and pulses generating elaborate three-dimensional patterns. Importantly, these patterns would not be random but entail recurring motifs in space and time: maybe a hotspot of red cells sending out a green 5-armed spiral, that then resolves into 5 new red foci separated by blue bars. It is these recurring patterns that the concept of serial homology via parts-as-processes seeks to capture.

Although pattern recognition is something that humans are extremely good at, it does not seem to me sufficient to let serial homology rest solely on the perception of a repeated pattern. After all, humans sometimes see patterns that are not real, for example constellations. Also, we would have no recourse for resolving an argument as to whether, for example, certain spines are serially homologous to the leaves or the shoots of the same plant (or both, if we allow for partial homology).

I propose that two structures on a single plant be judged serially homologous if there is some phene (structural or functional) of which they are both parts, where at least one of the phene's delimiting genetic factors controls the state of a GRN that is triggered discontinuously at different times or places during developing. For example, suppose that the production of all leaves requires a certain gene, $L$. That is to say, $L$ is one of the causal genetic factors underlying the leaf structural phene. If $L$ 
were expressed in patches of cells at different times and places within a developing plant, then we would have grounds for considering the parts of the phene developing from these patches as serial homologues under the umbrella phene 'leaf'. Thus, the hypothesis that there are multiple discrete 'leaves' caused by the expression of gene $L$ can be reframed as a counterfactual: If, $L$ had not been expressed in a certain patch of cells, would the plant be identical except for lacking the single 'leaf' derived from that patch?

Although there is certainly more work to be done to unpack the notion of repeated parts within an organism, I am confident that a rigorous treatment of serial homology can be achieved by combining part individuation at the whole-organism level, whether based on structures or functions, with the modularity of developmental processes. Such a rigorous framing of part-as-process has the potential to justify the claim that there is not just one umbrella phene 'leaf', but some specific and countable number of modular leaf instances on a particular plant — and likewise for phytomers, flowers, stamens, etc. Firming up such a part-as-process conception is an important endeavour, which resonates with the goals of developmental geneticists to understand the proximate causation of plant parts, while also providing morphologists and systematists a conceptual justification for the recognition of modular units within most plant bodies.

\section{Conclusions}

One of David Mabberley's legacies is a set of students who are reluctant to mindlessly fragment plants into a set of discrete parts, each assignable to one of a relatively small number of kinds. And a second accomplishment, I would claim, was to train botanists who would respond by doing more than throwing their hands up in frustration. I, hope, therefore, that this paper is one to which he would give a tolerable grade. Rather than provide one answer to a single question, "What is a part?", I have attempted to separate out multiple overlapping puzzles: What does it mean to assert that a single organism is composed of discrete developmental or selective parts? When can we claim that the parts in different organisms/species are homologous? And, finally, what are we implying when we claim that a 'part' recurs repeatedly during development? While I will not claim to have definitively answered these questions, I hope that the attempt was illuminating and raised some interesting biological and philosophical questions. At the very least, I hope the reader will have picked up my answer to the question posed in the title: depending on the precise framing of the question, plant parts can properly be considered structures, functions, and processes.

ACKNOWLEDGEMENTS. I thank Alistair Hay, Mark Olson, and one anonymous reviewer for helpful discussions and feedback on this paper. I also wish to express my sincere gratitude to Prof. David Mabberley for everything he taught me when I was an undergraduate, for his passionate teaching about the ecology and evolution of tropical plants, and for his great gift of instilling self-confidence (deserved or otherwise) in his tutees. 


\section{References}

Arber, A. (1950). The natural philosophy of plant form. Cambridge: Cambridge University Press.

Baum, D.A. (1998). Individuality and the existence of species through time. Syst. Biol. 47: 641-653.

Baum, D.A. (2013). Developmental causation and the problem of homology. Philos. Theory Biol. 5(201306): 1-14.

Baum, D.A. \& Donoghue, M.J. (2002). Transference of function, heterotopy, and the evolution of plant development. In: Cronk, Q.C.B., Bateman, R.A. \& Hawkins, J.A. (eds) Developmental genetics and plant evolution, pp. 52-69. London: Taylor \& Francis.

Beldade, P., Koops, K. \& Brakefield, P.M. (2002). Developmental constraints versus flexibility in morphological evolution. Nature 416: 844-847.

Bonner, J.T. (1988). The evolution of complexity by means of natural selection. Princeton University Press.

Cheverud, J.M. (1984). Quantitative genetics and developmental constraints on evolution by selection. J. Theor. Biol. 110: 155-171.

Claßen-Bockhoff, R. (2001). Plant morphology: the historic concepts of Wilhelm Troll, Walter Zimmermann and Agnes Arber. Ann. Bot. 88: 1153-1172.

Corner, E.J.H. (1958). Transference of function. Biol. J. Linn. Soc. 56: 33-40.

Ereshefsky, M. (1992). Eliminative pluralism. Philos. Sci. 59: 671-690.

Gould, S.J. (1977). Ontogeny and phylogeny. Harvard University Press.

Haag, E.S. (2007). Compensatory vs. pseudocompensatory evolution in molecular and developmental interactions. Genetica 129: 45-55.

Hay, A. (2019). Durianology, discovery, and saltation - the evolution of aroids. Gard. Bull. Singapore 71 (Suppl. 2): 259-316.

Hay, A. \& Mabberley, D.J. (1991). 'Transference of function' and the origin of aroids: their significance in early angiosperm evolution. Bot. Jahrb. Syst. 113: 339-428.

Hay, A. \& Mabberley, D.J. (1994). On perception of plant morphology: some implications for phylogeny. In: Ingram, D.S. \& Hudson, A. (eds) Shape and form in plants and fungi, Linnean Society Symposium Series No. 16, pp. 101-117. London: Academic Press.

Jong, K. \& Burtt, B.L. (1975). The evolution of morphological novelty exemplified in growthpatterns of some Gesneriaceae. New Phytol. 75: 297-311.

Kirchoff, B.K., Pfeifer, E. \& Rutishauser, R. (2008). Plant structure ontology: How should we label plant structures with doubtful or mixed identities? Zootaxa 1950: 103-122.

Lacroix, C., Jeune, B. \& Purcell-MacDonald, S. (2003). Shoot and compound leaf comparisons in eudicots: dynamic morphology as an alternative approach. Bot. J. Linn. Soc. 143: 219-230.

Mabberley, D.J. (1979). The species of Chisocheton (Meliaceae). Bull. Brit. Mus. (Nat. Hist.), Bot. 6: 301-386.

Mabberley, D.J. \& Hay, A. (1994). Homoeosis, canalization, decanalization, 'characters' and angiosperm origins. Edinburgh J. Bot. 51: 117-126.

Minelli, A. (2003). The origin and evolution of appendages. Int. J. Developm. Biol. 47: 573581.

Olson, M.E. (2019). Spandrels and trait delimitation: No such thing as "architectural constraint". Evol. Developm. 21: 59-71.

Olson, M.E. \& Rosell, J.A. (2006). Using heterochrony to detect modularity in the evolution of stem diversity in the plant family Moringaceae. Evolution 60: 724-734. 
Raff, E.C. \& Raff, R.A. (2000). Dissociability, modularity, evolvability. Evol. Developm. 2: $235-237$.

Reydon, T.A.C. (2005). On the nature of the species problem and the four meanings of 'species'. Stud. Hist. Philos. Sci. 36: 135-158.

Rutishauser, R. (1995). Developmental patterns of leaves in Podostemaceae compared with more typical flowering plants - saltational evolution and fuzzy morphology. Canad. J. Bot. 73: 1305-1317.

Rutishauser, R. (1997). Structural and developmental diversity in Podostemaceae (riverweeds). Aquatic Bot. 57: 29-70.

Rutishauser, R. (1999). Polymerous leaf whorls in vascular plants: developmental morphology and fuzziness of organ identities. Int. J. Pl. Sci. 160: S81-S103.

Rutishauser, R. (2016). Evolution of unusual morphologies in Lentibulariaceae (bladderworts and allies) and Podostemaceae (river-weeds): a pictorial report at the interface of developmental biology and morphological diversification. Ann. Bot. 117: 811-832.

Rutishauser, R. (2018). Ever since Darwin: why plants are important for evo-devo research. In: Fusco, G. (ed.) Perspectives on evolutionary and developmental biology: essays for Alessandro Minelli, pp. 41-55, Padova: Padova University Press.

Rutishauser, R. \& Isler, B. (2001). Developmental genetics and morphological evolution of flowering plants, especially bladderworts (Utricularia): Fuzzy arberian morphology complements classical morphology. Ann. Bot. 88: 1173-1202.

Sattler, R. (1984). Homology - a continuing challenge. Syst. Bot. 9: 382-394.

Sattler, R. (1988). Homeosis in plants. Amer. J. Bot. 75: 1606-1617.

Sattler, R. (1990). Towards a more dynamic plant morphology. Acta Biotheor. 38: 303-315.

Sattler, R. (1992). Process morphology: structural dynamics in development and evolution. Canad. J. Bot. 70: 708-714.

Sattler, R. (1996). Classical morphology and continuum morphology: opposition and continuum. Ann. Bot. 78: 577-581.

Sattler, R. \& Rutishauser, R. (1990). Structural and dynamic descriptions of the development of Utricularia foliosa and U. australis. Canad. J. Bot. 68: 1989-2003.

Sattler, R. \& Rutishauser, R. (1997). The fundamental relevance of morphology and morphogenesis to plant research. Ann. Bot. 80: 571-582.

Seibt, J. (2012). Process philosophy. In: Zalta, E.N. (ed.) The Stanford Encyclopedia of Philosophy (Winter 2018 Edition). https://plato.stanford.edu/entries/processphilosophy/

True, J.R. \& Haag, E.S. (2001). Developmental system drift and flexibility in evolutionary trajectories. Evol. Developm. 3: 109-119.

Wagner, G.P. (1989). The biological homology concept. Annual Rev. Ecol. Syst. 20: 51-69.

Wagner, G.P. (2007). The developmental genetics of homology. Nature Reviews Genetics 8: 473-479.

Wagner, G.P. \& Altenberg, L. (1996). Perspective: complex adaptations and the evolution of evolvability. Evolution 50: 967-976.

White, J. (1979). The plant as a metapopulation. Annual Rev. Ecol. Syst. 10: 109-145. 